\title{
DIRECT NUMERICAL SIMULATION OF A SELF-SIMILAR ADVERSE PRESSURE GRADIENT TURBULENT BOUNDARY LAYER
}

\author{
Vassili Kitsios, Callum Atkinson \\ Laboratory For Turbulence Research in Aerospace and Combustion, \\ Department of Mechanical and Aerospace Engineering, Monash University, Clayton 3800, AUSTRALIA \\ vassili.kitsios@monash.edu
}

Juan A. Sillero, Guillem Borrell, Javier Jiménez

School of Aeronautics, Universidad Politécnica de Madrid, E-28040 Madrid, SPAIN

\author{
Ayse Gul Gungor \\ Istanbul Technical University, \\ Department of Astronautical Engineering, Maslak 34469 Istanbul, TURKEY
}

\author{
Julio Soria \\ Laboratory For Turbulence Research in Aerospace and Combustion, \\ Department of Mechanical and Aerospace Engineering, Monash University, Clayton 3800, AUSTRALIA \\ Department of Aeronautical Engineering, King Abdulaziz University,
} Jeddah 21589, KINGDOM OF SAUDI ARABIA

\begin{abstract}
The statistical scaling properties of a self-similar adverse pressure gradient (APG) turbulent boundary layer (TBL) are presented. The intended flow is generated using the direct numerical simulation (DNS) TBL code of Simens et al. (2009) and Borrell et al. (2013), with a modified farfield boundary condition (BC). The conditions for self-similarity and appropriate scaling are derived, with mean and Reynolds stress profiles presented using this scaling. The APG and ZPG DNS are also compared under the classical viscous scaling.
\end{abstract}

\section{INTRODUCTION}

The performance of many engineering systems relies on fluid flows remaining attached to aerodynamic surfaces, with flow separation potentially resulting in catastrophic consequences or at best energy efficiency degradation. The study of fluid flow separation is, therefore, of utmost importance. Real world examples include the flow over aerofoil geometries such as aircraft wings, wind turbine blades, and turbo-machinery. These configurations are difficult to systematically study as the pressure gradient is constantly changing in the streamwise direction, as in the large eddy simulation of Kitsios et al. (2011).

Here we study the canonical self-similar APG TBL. A self-similar TBL is defined as each of the terms in the Navier-Stokes equations having the same proportionality with the streamwise position (Castillo \& Wang, 2004) Here we undertake simulations of flow over a flat surface using the TBL DNS code of Simens et al. (2009) and
Borrell et al. (2013), with a modified farfield APG boundary condition (BC). This effectively decouples the effect of upstream flow history and surface curvature from the influence of the local pressure gradient. Self-similarity is demonstrated in the present APG TBL over a momentum thickness based Reynolds number range of $R e_{\delta_{2}} \approx 3000$ to 5000. Previous DNS of TBL APG include the non-selfsimilar separated flow of Gungor et al. (2012), and selfsimilar APG cases at relatively low Reynolds numbers in Lee \& Sung (2008)

An overview of the original ZPG TBL DNS code is first presented. Following this the modifications to the $\mathrm{BC}$ required to generate the self-similar APG TBL are detailed. The conditions for self-similarity and appropriate scaling are derived from first principles, and are evaluated for both the APG and ZPG cases. Profiles of the mean velocity deficit and Reynolds stresses from the DNS of the APG are then compared to those of the ZPG DNS on the basis of viscous scaling. The APG DNS profiles are also scaled consistent with the results from self-similarity analysis.

\section{DIRECT NUMERICAL SIMULATION}

The TBL DNS code adopted within solves the NavierStokes equations in a three-dimensional rectangular volume, with constant density $(\rho)$ and kinematic viscosity $(v)$. The three flow directions are the streamwise $(x)$, wall-normal $(y)$ and spanwise $(z)$, with respective instantaneous velocity components in these directions of $U, V$ and $W$. Throughout the paper the time averaged velocity components are represented by $(\langle U\rangle,\langle V\rangle,\langle W\rangle) \equiv(\bar{u}, \bar{v}, \bar{w})$, 


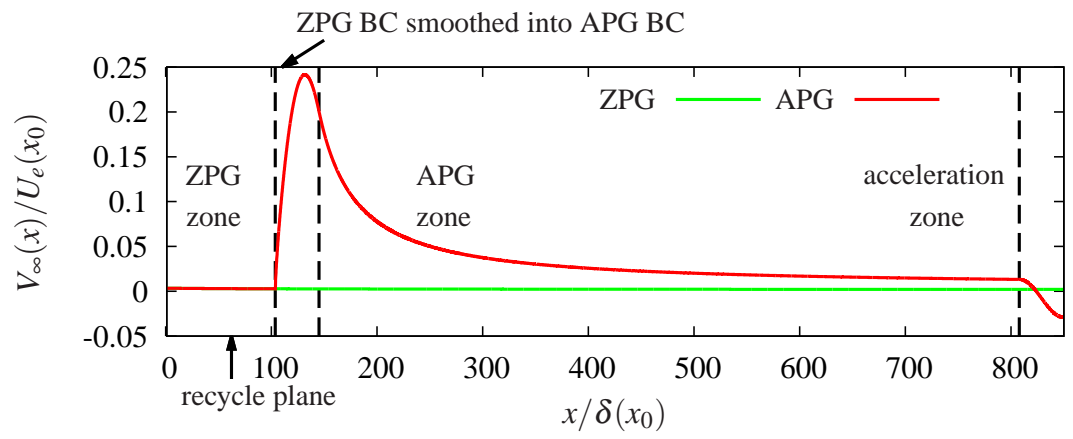

Figure 1. Farfield wall normal velocity BC in the APG (red line) and ZPG (green line) DNS.

with associated fluctuating components of $(u, v, w)$, where the brackets $\langle\cdot\rangle$ denote the time averaging operation. A fractional-step method is used to solve the governing equations for the velocity and pressure $(P)$ fields (Harlow \& Welch, 1965; Perot, 1993). Fourier decomposition is used in the periodic spanwise direction, with compact finite difference in the aperiodic wall-normal and streamwise directions (Lele, 1992). The equations are stepped forward in time using a modified three sub-step Runge-Kutta scheme (Simens et al., 2009). The code utilises MPI parallelisation to decompose the domain in $x$, and openMP parallelisation to decompose in $y$. For further details on the message passing refer to (Borrell et al., 2013).

The boundary conditions of the ZPG TBL DNS code are as follows. The bottom surface is a flat plate with a noslip (zero velocity) BC. The spanwise boundaries are periodic. Due to the TBL growing in height as it develops in the streamwise direction, a downstream streamwise normal recycling plane is copied, and mapped to the inlet $\mathrm{BC}$ (Sillero et al., 2013). At the farfield boundary the spanwise vorticity is zero, and the wall normal velocity is

$$
V_{Z P G}(x)=U_{Z P G} \partial_{x} \delta_{1}(x)
$$

where $U_{Z P G}$ is the constant freestream streamwise velocity, $\delta_{1}$ is the displacement thickness (Sillero, 2014). Throughout the document $\partial_{x} \equiv \partial / \partial x$ and also $\partial_{y} \equiv \partial / \partial y$.

To generate the self-similar APG TBL flow, the farfield wall-normal velocity BC must be modified. From Mellor \& Gibson (1966) the freestream streamwise velocity $U_{A P G}(x) \propto x^{m}$, where $m=-0.23$ for the incipient separation APG TBL with the wall shear stress $\left(\tau_{w}\right)$ approaches zero. The wall normal suction velocity $V_{A P G}(x)$ is deduced from $U_{A P G}(x)$ via the boundary layer streamfunction solution in the farfield region to be

$$
V_{A P G}(x)=-\left[y_{\infty}-\delta_{1}\right] \partial_{x} U_{A P G}+U_{A P G} \partial_{x} \delta_{1},
$$

where $y_{\infty}$ is the wall normal position of the farfield boundary (Mellor \& Gibson, 1966). This can also be deduced from the similarity analysis of continuity equation in the following section.

The structure of the complete farfield wall normal BC, $V_{\infty}(x)$, is as follows. In the APG TBL DNS, to allow the rescaling necessary for the inlet BC an initial ZPG TBL is simulated up until the streamwise position $x_{S}=100 \delta\left(x_{0}\right)$ (located after the recycling plane) by applying $V_{Z P G}(x)$ as defined in (1). Note $\delta\left(x_{0}\right)$ is the boundary layer thickness (point of maximum velocity, $U_{e}$, along the profile) at the inlet of position $x=x_{0}$. Downstream of the position $x_{f}=140 \delta\left(x_{0}\right)$ the wall normal velocity $V_{A P G}(x)$ is applied at the farfield boundary as given by (2), which imparts the desired deceleration and hence expansion of the boundary layer. From $x_{s}$ to $x_{f}$ the velocity $V_{A P G}(x)$ is gradually introduced using a smoothing function. Finally the farfield velocity is transitioned from suction $\left(V_{\infty}(x)>0\right)$ at $x_{b}=760 \delta\left(x_{0}\right)$ to blowing $\left(V_{\infty}(x)<0\right)$ at the outlet to reduce the number of instantaneous reversed flow events, such that numerical stability of the outflow boundary condition is maintained. The ZPG and APG farfield boundary conditions, $U(x) / U_{\infty}\left(x_{0}\right)$, is illustrated in Fig. 1 , where $U_{\infty}\left(x_{0}\right)$ is the freestream streamwise velocity at the inlet.

The streamwise, wall normal and spanwise domain extents are $\left(L_{x}, L_{y}, L_{z}\right) / \delta\left(x_{0}\right)=(801,38,134)$ for the ZPG TBL DNS and $\left(L_{x}, L_{y}, L_{z}\right) / \delta\left(x_{0}\right)=(801,70,134)$ for the APG case. The associated number of grid points are $N_{x} \times N_{y} \times N_{z}=8193 \times 315 \times 1362$ for the ZPG and $N_{x} \times$ $N_{y} \times N_{z}=8193 \times 500 \times 1362$ for the APG. The APG simulation has a larger wall normal domain $\left(L_{y}\right)$ and more points in this direction $\left(N_{y}\right)$ due to the APG TBL expanding more quickly in the streamwise direction than the ZPG TBL. Both simulations have the same grid spacings of $\left(\Delta x, \Delta y_{\text {wall }}, \Delta y_{\infty}, \Delta z\right) / \delta\left(x_{0}\right)=(0.098,0.0026,0.17,0.098)$, where $\Delta x$ and $\Delta z$ are the constant spacing in the streamwise and spanwise directions, with $\Delta y_{\text {wall }}$ and $\Delta y_{\infty}$ the wall normal grid spacing at the wall and at the farfield boundary receptively. In both simulations the Courant number was set to unity. The length scales are non-dimensionalised by $\delta\left(x_{0}\right)$ as viscous scaling is inappropriate for APG flows.

The ZPG and APG boundary layer properties are now characterised within the zone of interest from $x=300 \delta\left(x_{0}\right)$ to $x=630 \delta\left(x_{0}\right)$. In all of the following figures the green and red lines represent the ZPG and APG cases respectively. The momentum thickness $\left(\delta_{2}\right)$ based Reynolds number $\left(R e_{\delta_{2}}\right)$ illustrated in Fig. 2(a), increases in the APG TBL more rapidly than the ZPG TBL. This is because the APG TBL expands more rapidly than the ZPG TBL in the streamwise direction as illustrated by $\delta, \delta_{1}$ and $\delta_{2}$ in Fig. 2(b), Fig. 2(c) and Fig. 2(d) respectively. The shape factor $H=\delta_{1} / \delta_{2}$ is illustrated in Fig. 2(e) and is constant over this range. The APG TBL is additionally decelerated via the $\mathrm{BC}$ as illustrated in the reduction of the outer reference velocity $\left(U_{e}\right)$ in Fig. 2(f). The expansion of the boundary layer coincides with a reduction of the wall shear stress $\left(\tau_{w}\right)$. In Fig. $2(\mathrm{~g}), \tau_{w}$ of the APG case is less than that of ZPG TBL as the former is decelerated more than the latter, which is also evident in the skin friction coefficient $\left(C_{f}\right)$ illustrated in Fig. 2(h). The parameter $\beta=\delta_{1}\left(\partial_{x} P_{e}\right) / \tau_{w}$ quantifies the strength of the pressure gradient and is illus- 
(a)

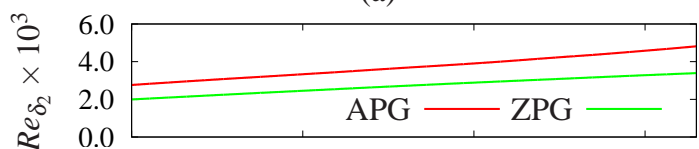

(b)

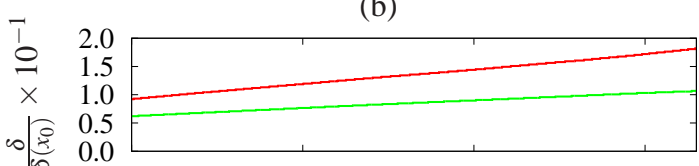

(c)

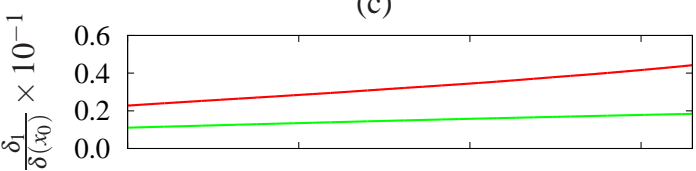

(d)

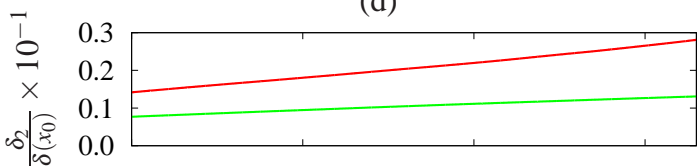

(e)

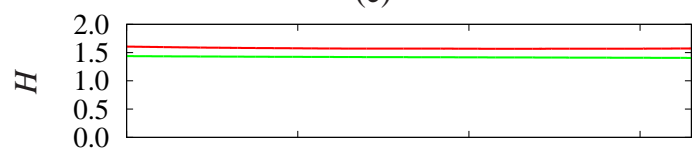

(f)

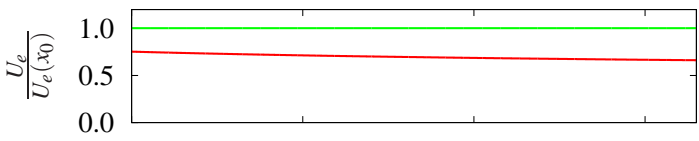

$(\mathrm{g})$

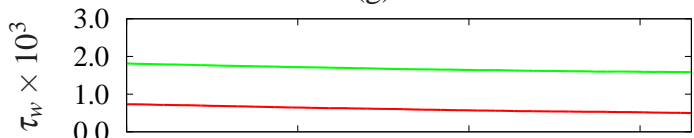

(h)

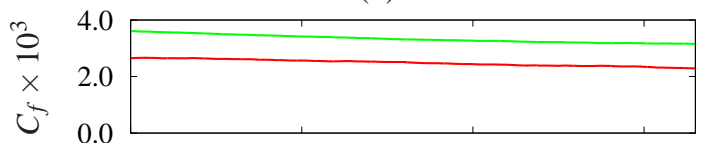

(i)

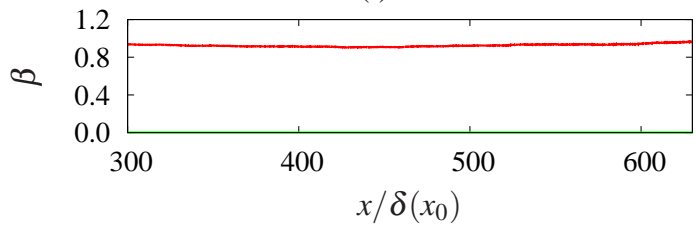

Figure 2. Boundary layer properties of the APG and ZPG DNS: (a) momentum thickness Reynolds number, $R e_{\delta_{2}}=$ $U_{e} \delta_{2} / v$; (b) boundary layer thickness, $\delta$; (c) displacement thickness, $\delta_{1}$; (d) momentum thickness, $\delta_{2}$; (e) shape factor, $H=\delta_{1} / \delta_{2}$; (f) outer reference velocity, $U_{e}$; (g) wall shear stress, $\tau_{w}$; and (h) skin friction coefficient, $C_{f}=2 \tau_{w} / U_{e}^{2}$; and (i) pressure gradient parameter, $\beta=\delta_{1}\left(\partial_{x} P_{e}\right) / \tau_{w}$, with the horizontal axis applicable to all figures, and $\beta=0$ for the ZPG TBL.

trated in Fig. 2(i). For the incipient separation case $\tau_{w}$ (and $C_{f}$ ) approaches zero, and hence $\beta \rightarrow \infty$. The present APG TBL DNS has not yet attained the desired state, however, an additional DNS is currently being run with a stronger pressure gradient, producing a lower $C_{f}$ and hence larger $\beta$.

\section{SIMILARITY CONDITIONS}

To achieve a self-similar boundary layer there are various quantities that must be independent of $x$. These quantities are derived from first principles following the analysis of Castillo \& Wang (2004), which are then evaluated for the present data. We start with the Reynolds averaged Navier-Stokes continuity and streamwise momentum equations specific for boundary layer layers given by

$$
\begin{aligned}
\partial_{x} \bar{u}+\partial_{y} \bar{v} & =0, \text { and } \\
\bar{u} \partial_{x} \bar{u}+\bar{v} \partial_{y} \bar{u} & =-\left(\partial_{x} P_{e}\right) / \rho+\partial_{x}\left\langle v^{2}\right\rangle \\
& -\partial_{x}\left\langle u^{2}\right\rangle-\partial_{y}\langle u v\rangle+v \partial_{y} \partial_{y} \bar{u},
\end{aligned}
$$

respectively, where $v$ is the kinetic viscosity, and the farfield pressure gradient $\partial_{x} P_{e}=-\rho U_{e} \partial_{x} U_{e}$. A derivation of these equations from the complete instantaneous Navier-Stokes can be found in Pope (2008). The following general scaling for the mean field and Reynolds stresses is adopted with

$$
\begin{aligned}
\bar{u}(x, y) & =U_{e}(x)+U_{0}(x) f(\zeta), \\
\langle u v\rangle(x, y) & =-R_{u v}(x) r_{u v}(\zeta), \\
\left\langle u^{2}\right\rangle(x, y) & =R_{u u}(x) r_{u u}(\zeta), \\
\left\langle v^{2}\right\rangle(x, y) & =R_{v v}(x) r_{v v}(\zeta), \text { and } \\
\zeta & =y / L_{0}(x), \text { where } \\
L_{0}(x) & =\delta_{1}(x) U_{e}(x) / U_{0}(x) .
\end{aligned}
$$

Recall $U_{e}$ is the maximum velocity along the profile, $U_{0}$ is the velocity scale used to nondimensionalise the velocity deficit, and $\delta_{1}$ is the displacement thickness defined by

$$
\delta_{1}(x)=\int_{0}^{\delta}\left(1-U(x, y) / U_{e}(x)\right) d y,
$$

with $\delta$ the wall normal position of $U_{e}$. This definition for $\zeta$ ensures that

$$
\int_{0}^{\delta / L_{0}} f(\zeta) d \zeta=-1
$$

Likewise the integrals from $\zeta=0$ to $\zeta=\delta / L_{0}$ of the similarity functions for the Reynolds stresses $r_{u v}(\zeta), r_{u и}(\zeta)$ and $r_{v v}(\zeta)$ are all defined to be equal to 1 . This means the functions $R_{u v}(x), R_{u u}(x)$, and $R_{v v}(x)$ can be determined at each $x$ position from the wall normal integrals of $-\langle u v\rangle(x, y)$, $\left\langle u^{2}\right\rangle(x, y)$ and $\left\langle v^{2}\right\rangle(x, y)$ respectively.

By substituting (5) to (8) into the continuity equation (3), applying the chain rule and integration by parts, we get the following expression for the mean wall normal velocity

$$
\begin{gathered}
\bar{v}=-\zeta L_{0} \partial_{x} U_{e}-F \partial_{x}\left(U_{0} L_{0}\right)+f \zeta U_{0} \partial_{x} L_{0}, \text { with (13) } \\
F(\zeta)=\int_{0}^{\zeta} f(\tilde{\zeta}) d \tilde{\zeta} .
\end{gathered}
$$

Making the same substitutions into the momentum equation (4), along with (13) for $\bar{v}$, and grouping like terms produces

$$
\begin{aligned}
& {\left[U_{0} \partial_{x} U_{e}+U_{e} \partial_{x} U_{0}\right] f+\left[U_{0} \partial_{x} U_{0}\right] f^{2} } \\
- & {\left[U_{0} U_{e} / L_{1}+U_{0} \partial_{x} U_{e}\right] \zeta f^{\prime}-\left[U_{0}^{2} / L_{1}+U_{0} \partial_{x} U_{0}\right] F f^{\prime} } \\
= & -\left[R_{v v} / L_{1}\right] r_{v v}^{\prime} \zeta+\left[R_{u u} / L_{1}\right] r_{u u}^{\prime} \zeta+\left[R_{u v} / L_{0}\right] r_{u v}^{\prime} \\
+ & {\left[\partial_{x} R_{v v}\right] r_{v v}-\left[\partial_{x} R_{u u}\right] r_{u u}+\left[v U_{0} / L_{0}^{2}\right] f^{\prime \prime}, }
\end{aligned}
$$


where $L_{1}=L_{0}^{-1} \partial_{x} L_{0}$, and the superscript ' denotes $\partial / \partial \zeta$. The terms in the square brackets in (15) are a function of only $x$, and the remaining terms are functions of only $\zeta$.

For a boundary layer to be self-similar each of the terms in the square brackets of (15) must all have the same proportionality with $x$. By inspecting the first three terms in (15) we find that $U_{0} \partial_{x} U_{e} \propto U_{e} \partial_{x} U_{0} \propto U_{0} \partial_{x} U_{0}$, which can call only be satisfied if

$$
U_{0}=K U_{e},
$$

where $K$ is an arbitrary constant. This simplifies the definition of the length scale in (10) to $L_{0}=\delta_{1} U_{e} / U_{0}=\delta_{1} / K$. Substituting in these relationships for $U_{e}$ and $L_{0}$ into (15) and dividing through by $-U_{0}^{2}\left(\partial_{x} \delta_{1}\right) / \delta_{1}$ produces

$$
\begin{aligned}
\frac{2}{K} \Lambda f & +\Lambda f^{2}-\frac{1}{K}[\Lambda-1] \zeta f^{\prime}-[\Lambda-1] F f^{\prime} \\
& =C_{v v} r_{v v}^{\prime} \zeta-C_{u u} r_{u u}^{\prime} \zeta-K C_{u v} r_{u v}^{\prime} \\
& -D_{v v} r_{v v}+D_{u u} r_{u u}-K^{2} C_{v} f^{\prime \prime}, \text { where } \\
C_{u u} & =R_{u u} / U_{0}^{2}, \\
C_{v v} & =R_{v v} / U_{0}^{2}, \\
C_{u v} & =R_{u v} /\left(U_{0}^{2} \partial_{x} \delta_{1}\right), \\
D_{u u} & =\partial_{x} R_{u u} \delta_{1} /\left(U_{0}^{2} \partial_{x} \delta_{1}\right), \\
D_{v v} & =\partial_{x} R_{v v} \delta_{1} /\left(U_{0}^{2} \partial_{x} \delta_{1}\right), \\
C_{v} & =-v /\left(U_{0} \delta_{1} \partial_{x} \delta_{1}\right), \text { and } \\
\Lambda & =-\delta_{1} U_{0} \partial_{x} U_{0} /\left(U_{0}^{2} \partial_{x} \delta_{1}\right)=-\delta_{1} U_{e} \partial_{x} U_{e} /\left(U_{e}^{2} \partial_{x} \delta_{1}\right) \\
& =\delta_{1} \partial_{x} P_{e} /\left(\rho U_{e}^{2} \partial_{x} \delta_{1}\right)=\left(U_{p} / U_{e}\right)^{2} /\left(\partial_{x} \delta_{1}\right),
\end{aligned}
$$

with constants independent of $x$ for self-similar TBL. The pressure velocity $U_{p}=\sqrt{\left(\partial_{x} P_{e}\right) \delta_{1} / \rho}$ (Mellor \& Gibson, 1966), and $\Lambda$ is as defined in Castillo \& Wang (2004).

We can also determine a relationship between the freestream velocity and the displacement thickness by rearranging the definition of $\Lambda$ in (24) into the form

$$
-\Lambda\left(\partial_{x} \delta_{1}\right) / \delta_{1}=\left(\partial_{x} U_{e}\right) / U_{e}
$$

Integrating both sides with respect to $x$ produces

$$
U_{e}=A \delta_{1}^{-\Lambda}
$$

where $A$ is a constant of integration. In Fig. 3 we have plotted the natural $\log$ of $U_{e}$ against the natural $\log$ of $\delta_{1}$, the gradient of which should be $-\Lambda$. For the case of a linearly increasing displacement thickness $-\Lambda=m$.

It can also be shown that if $\Lambda, C_{u u}$ and $C_{v v}$ are independent of $x$, then $D_{u u}$ and $D_{v v}$ must also be independent of $x$. There are, therefore, only five unique coefficients that need to be independent of $x$ to ensure self-similarity, which are $\Lambda$, $C_{u v}, C_{u u}, C_{v v}$ and $C_{v}$. These coefficients are evaluated for the APG and ZPG cases in Fig. 4, with the APG coefficients relatively independent of $x$, with expection of perhaps $C_{V}$.

\section{Scaling of the statistical profiles}

Mean streamwise velocity deficit profiles $\left(U_{e}-\bar{u}\right)$ are now presented at the streamwise locations indicated by the

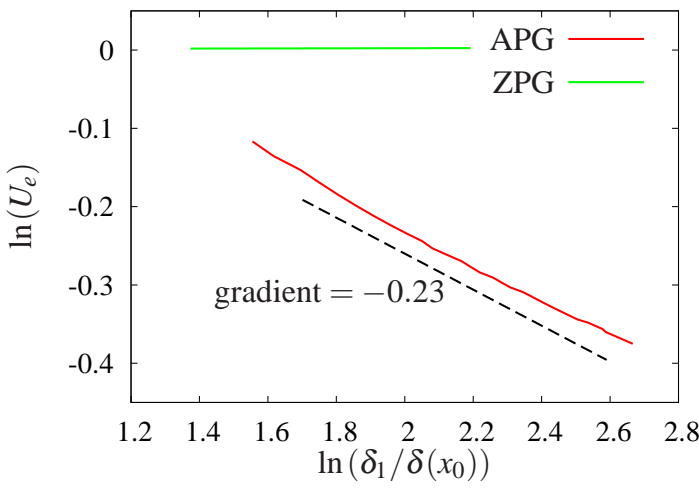

Figure 3. Natural $\log$ of outer reference velocity, $U_{e}$, as a function of the natural $\log$ of displacement thickness, $\delta_{1}$. Dashed black line has a reference gradient of -0.23 .

(a)

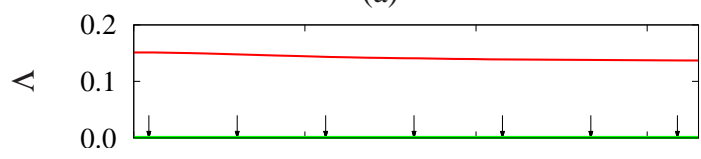

(b)

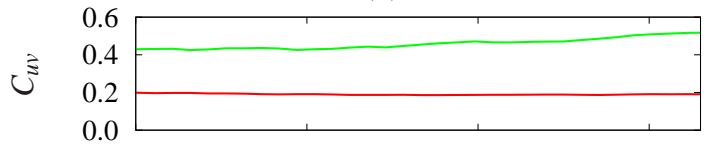

(c)

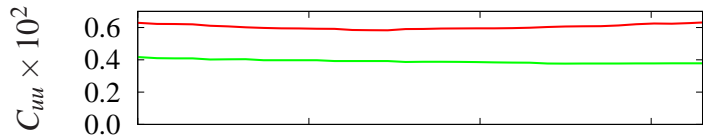

(d)

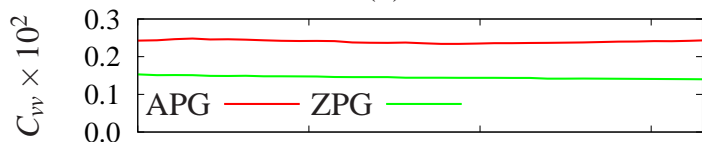

(e)

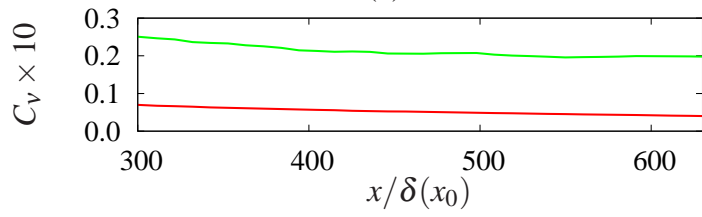

Figure 4. Coefficients to assess the self-similarity of the APG and ZPG TBL DNS on the basis of: (a) $\Lambda$, with arrows indicating the positions of the APG TBL velocity profiles illustrated in Fig. 5 and Fig. 6; (b) $C_{u v}$; (c) $C_{u u}$; (d) $C_{v v}$; and (e) $C_{v}$, with the horizontal axis applicable to all figures.

arrows in Fig. 4(a). In Fig. 5(a) the deficit profiles are nondimensionalised by the friction velocity $\left(u_{\tau}=\sqrt{\tau_{w} / \rho}\right)$ and plotted against $y / \delta$. The dots in this figure represent results from the previous ZPG DNS of Jiménez et al. (2010), which agree with the present ZPG simulation. When scaled by $u_{\tau}$, the non-dimensional velocity profiles near the wall increase in the downstream direction - indicated by the arrow - as $u_{\tau}$ decreases. However, when scaled by $U_{e}$ and $\delta_{1}$ (in line with the theory in the previous section) the profiles do collapse, as illustrated in Fig. 5(b). The solid black line 
(a) Viscous Scaling

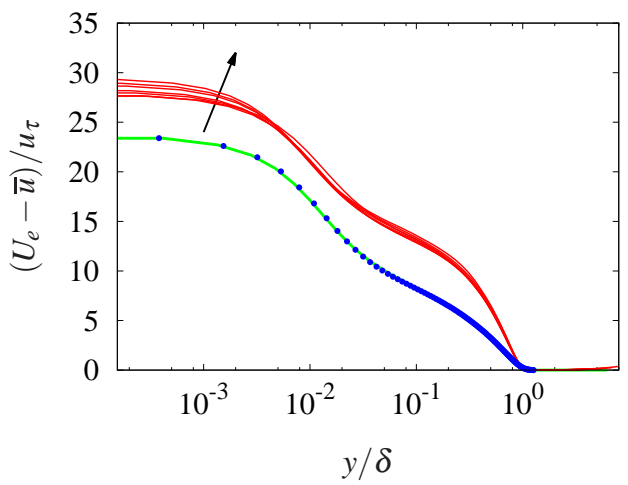

(b) Outer Scaling

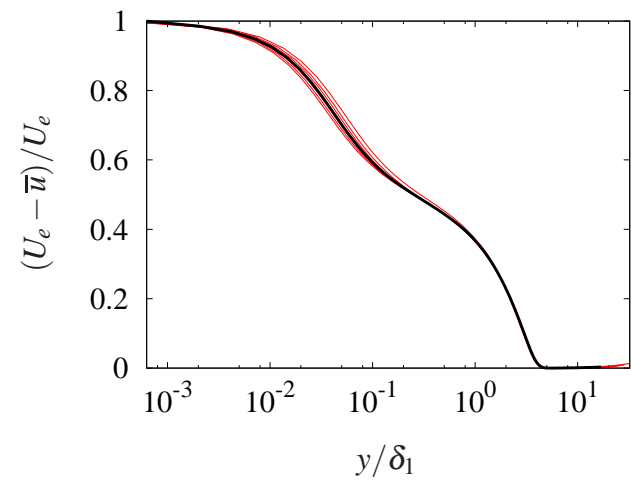

Figure 5. Mean velocity deficit profiles nondimensionalised by: (a) friction velocity, $u_{\tau}$, and boundary layer thickness, $\delta$; and (b) reference outer velocity, $U_{e}$, and displacement thickness, $\delta_{1}$. ZPG TBL DNS of Jiménez et al. (2010) - blue dots; ZPG TBL DNS current simulation - green line; APG TBL DNS from current simulation at different streamwise locations - red lines; streamwise averaged scaled profiles - black lines. Positions of the APG TBL profiles are illustrated in Fig. 4(a), with arrows in left column indicating the direction of increasing $x$ position.

represents the streamwise average in the scaled coordinates.

As undertaken for the velocity deficit profiles, the Reynolds stresses are now presented scaled on the basis of initially the wall shear stress and then on the basis of the outer flow (or effectively the pressure gradient). Under the former scaling the Reynolds stress profiles are nondimensionalised using a velocity scale of $u_{\tau}$ and length scale of $v / u_{\tau}$. Under the latter scaling the pertinent velocity and length scales are again $U_{e}$ and $\delta_{1}$. Profiles of $\langle u u\rangle$ are plotted in viscous scaling in Fig. 6(a), which again increases as $u_{\tau}$ decreases in the downstream direction. A second outer peak is also evident. Similar observations are also made concerning $\langle v v\rangle,\langle w w\rangle$, and $\langle u v\rangle$, plotted under viscous scaling in Fig. 6(c), Fig. 6(e), and Fig. 6(g) respectively. All of these Reynolds stresses increase in magnitude as $u_{\tau}$ decreases in the downstream direction, with a prominent second outer peak. The profiles collapse for all of the streamwise stations when plotted in outer scaling as illustrated for $\langle u u\rangle,\langle v v\rangle,\langle w w\rangle$ and $\langle u v\rangle$ in Fig. 6(b), Fig. 6(d), Fig. 6(f), and Fig. 6(h) respectively.

\section{CONCLUDING REMARKS}

An adverse pressure gradient turbulent boundary layer was generated via direct numerical simulation with a modified farfield boundary condition. The coefficients quantifying the extent of self-similarity are relatively constant for the adverse pressure gradient case over a momentum thickness based Reynolds number range of $R e_{\delta_{2}} \approx 3000$ to 5000 . Within this domain, mean velocity deficit and Reynolds stress profiles collapse under outer scaling. The Reynolds stresses also exhibit a second outer peak.

The authors would like to acknowledge the research funding from the Australian Research Council and European Research Council, and the computational resources provided by the Australian National Computational Infrastructure, iVEC and PRACE. Julio Soria gratefully acknowledges the support of an Australian Research Council Discovery Outstanding Researcher Award fellowship.

\section{REFERENCES}

Borrell, G., Sillero, J.A. \& Jiménez, J. 2013 A code for direct numerical simulation of turbulent boundary layers at high reynolds numbers in BG/P supercomputers. Comp. Fluids 80, 37-43.

Castillo, L. \& Wang, X. 2004 Similariy analysis for nonequilibrium turbulent boundary layers. J. Fluids Eng. 126, 827-834.

Gungor, A.G., Simens, M.P. \& Jiménez, J. 2012 Direct numerical simulation of wake-perturbed separated boundary layers. J. Turb 134, 061024.

Harlow, F.H. \& Welch, J.E. 1965 Numerical calculation of time-dependent viscous incompressible flow of fluid with free surface. Phys. Fluids $\mathbf{8}$ (12).

Jiménez, J., Hoyas, S., Simens, M.P. \& Mizuno, Y. 2010 Turbulent boundary layers and channels at moderate Reynolds numbers. J. Fluid. Mech. 657 (22), 335-360.

Kitsios, V., Cordier, L., Bonnet, J.-P., Ooi, A. \& Soria, J. 2011 On the coherent structures and stability properties of a leading edge separated airfoil with turbulent recirculation. J. Fluid Mech. 683, 395-416.

Lee, J.-H. \& Sung, J. 2008 Effects of an adverse pressure gradient on a turbulent boundary layer. Int. J. Heat Fluid Fl. 29, 568-578.

Lele, S.K. 1992 Compact finite difference schemes with spectral-like resolution. J. Comput. Phys. 103, 16-42.

Mellor, G.L. \& Gibson, D.M. 1966 Equilibrium turbulent boundary layers. J. Fluid. Mech. 24, 225-253.

Perot, J.B. 1993 An analysis of the fractional step method. J. Comput. Phys. 108, 51-58.

Pope, S. B. 2008 Turbulent flows. Cambridge University Press.

Sillero, J. 2014 High Reynolds number turbulent boundary layers. PhD thesis, Universidad Politénica de Madrid.

Sillero, J.A., Jiménez, J. \& Moser, R.D. 2013 One-point statistics for turbulent wall-bounded at Reynolds numbers up to $\delta^{+} \approx 2000$. Phys. Fluids 25, 105102.

Simens, M. P., Jiménez, J., Hoyas, S. \& Mizuno, Y. 2009 A high-resolution code for tubulent boundary layers. J. Comp. Phys. 228, 4128-4231. 


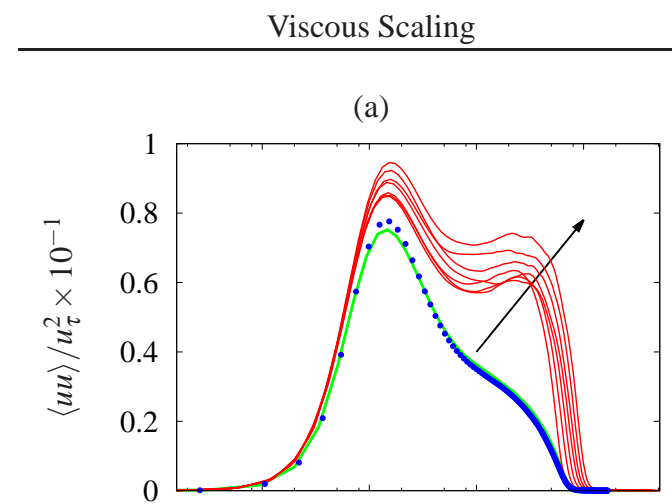

(c)

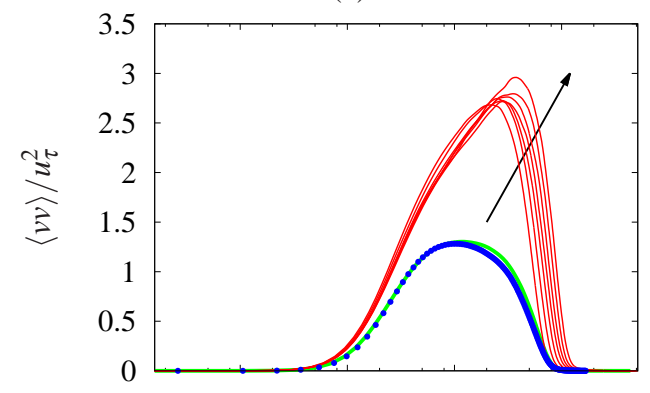

(e)

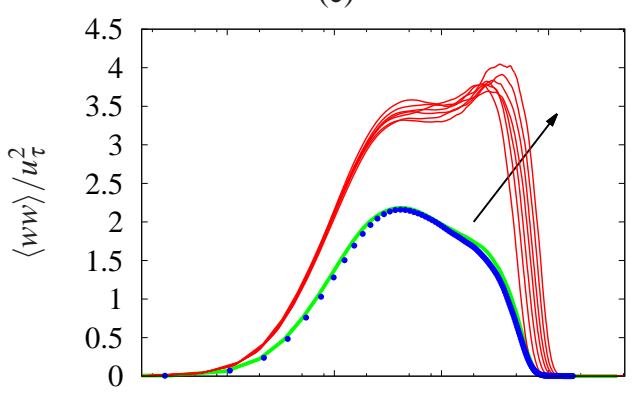

(g)

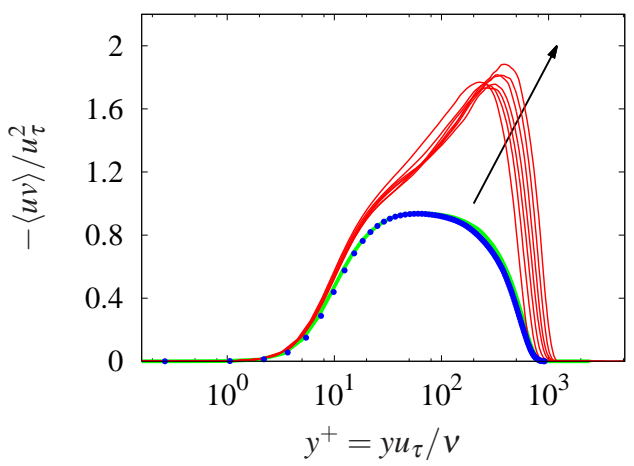

Outer Scaling

(b)

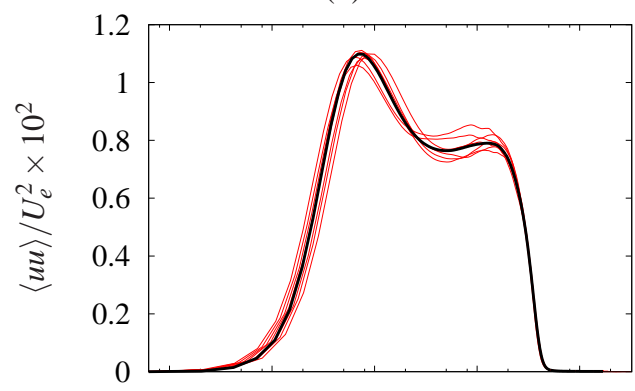

(d)

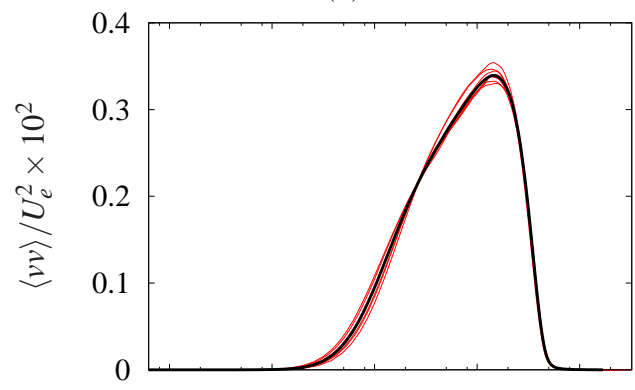

(f)

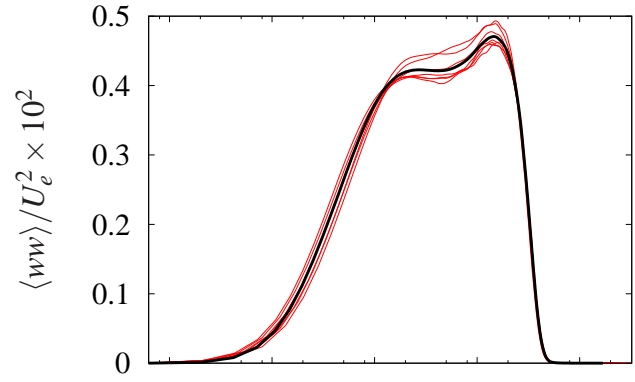

(h)

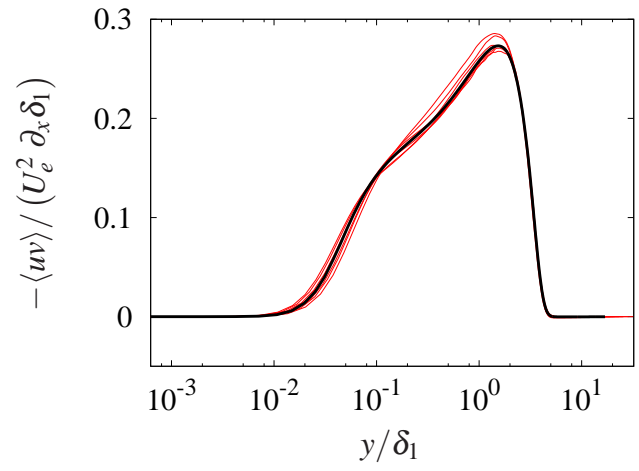

Figure 6. Reynolds stress profiles: (a) $\langle u u\rangle$ in viscous scaling nondimensionalised by friction velocity, $u_{\tau}$, and viscous length scale, $v / u_{\tau}$; (b) $\langle u u\rangle$ in outer scaling nondimensionalised by reference outer velocity, $U_{e}$, and displacement thickness, $\delta_{1}$; (c) $\langle v v\rangle$ in viscous scaling; (d) $\langle v v\rangle$ in outer scaling; (e) $\langle w w\rangle$ in viscous scaling; (f) $\langle w w\rangle$ in outer scaling; (g) $\langle u v\rangle$ in viscous scaling, with horizontal scale applicable to all plots in same column; and (h) $\langle u v\rangle$ in outer scaling, with horizontal scale applicable to all plots in same column. ZPG TBL DNS of Jiménez et al. (2010) - blue dots; ZPG TBL DNS current simulation - green line; APG TBL DNS from current simulation at different streamwise locations - red lines; streamwise averaged scaled profiles - black lines. Positions of the APG TBL profiles are illustrated in Fig. 4(a), with arrows in left column indicating the direction of increasing $x$ position. 